\title{
Adaptive Estimation of Heteroscedastic Money Demand Model of Pakistan
}

\author{
Muhammad Aslam \\ Department of Statistics \\ Bahauddin Zakariya University, Multan, Pakistan \\ E-mail: aslamasadi@bzu.edu.pk \\ G. R. Pasha \\ Department of Statistics \\ Bahauddin Zakariya University, Multan, Pakistan \\ E-mail: drpasha@bzu.edu.pk
}

\begin{abstract}
For the problem of estimation of Money demand model of Pakistan, money supply $\left(M_{1}\right)$ shows heteroscedasticity of the unknown form. For estimation of such model we compare two adaptive estimators with ordinary least squares estimator and show the attractive performance of the adaptive estimators, namely, nonparametric kernel estimator and nearest neighbour regression estimator. These comparisons are made on the basis standard errors of the estimated coefficients, standard error of regression, Akaike Information Criteria (AIC) value, and the Durban-Watson statistic for autocorrelation. We further show that nearest neighbour regression estimator performs better when comparing with the other nonparametric kernel estimator.
\end{abstract}

Keywords: Adaptive estimator; Heteroscedasticity; Money supply; Nonparametric kernel Estimator; Nearest neighbour regression estimator.

\section{Introduction}

In monetary sector, money supply is a major variable of interest. Money supply, a macroeconomic concept, is the quantity of money available within the economy to purchase goods, services, and securities. Money supply is divided into three categories--M1, M2, and M3--according to the type and size of account in which the instrument is kept. According to Ahmed et. al. (2005), there are several channels through which changes in money supply affects output. A few prominent channels are the interest rate channel, credit channel, exchange rate channel, and asset price channel. So money supply is important to economists trying to understand how policies will affect interest rates and growth. A large number of studies can be found, in this aspect, in available economic literature, for example, see Khan and Hussain (2005).

A variety of economic problems entirely rely on regression models. Incomeexpenditure models, demand-supply models, saving models, stock returns, market evaluations, etc. all rest, heavily, on such models. In recent economic literature, a number of studies can be found that rely on regression models while dealing with money supply as a variable of interest being function of many other monetary variables (see Hossain, 1994 and Gujarati, 2003, for example). 
The basic version of regression model assumes that the expected value of all error terms, when squared, is the same at any given point. This assumption is called homoscedasticity. If this assumption is not met, there is a mark of heteroscedasticity existence. In other words, the regression disturbances whose variances are not constant across observations are heteroscedastic. The most common framework in which heteroscedasticity is studied in econometrics is in the context of the linear regression models. While errors of measurements, sampling strategies, model misspecifications, and presence of outliers etc. are the main causes to introduce heteroscedasticity (see also Griffiths, 1999 and Gujarati, 2003 for more details).

In the presence of heteroscedasticity, ordinary least squares (OLS) does not result in biased and inconsistent parameter estimates. However, OLS estimates are no longer best linear unbiased estimators (BLUE). That is, among all the unbiased estimators, OLS does not provide the estimate with the smallest variance. In addition, the standard errors of the estimates become biased and inconsistent when heteroscedasticity is present. Thus, the conventional $t$ and $F$ test do not have their namesake distributions, even asymptotically under the null hypothesis that they test. This, in turn, leads to bias in test statistics and confidence intervals. Depending on the nature of the heteroscedasticity, significance tests can be too high or too low. These effects are not ignorable as earlier noted by Geary (1966), White (1980) and Pasha (1982) among many others.

In the present article, we estimate money supply model as a function of gross domestic product (GDP) of Pakistan. We take annual (1975 to 2003) available data from State Bank of Pakistan about Money Supply $\left(\mathrm{M}_{1}\right)$ and GDP. Unlike in the available studies, we do not brush aside the heterogeneity of the error terms but provide more efficient estimates using adaptive estimation procedures in the presence of heteroscedasticity.

In Section 2, we present the adaptive estimators; nonparametric kernel estimator proposed by Carroll (1982) and nearest neighbour regression (NNR) given by Robinson (1987). In Section 3, we formulate the money demand model and show the performances of the estimates while Section 4 reserves for conclusion.

\section{Adaptive Estimation}

When the form of heteroscedasticity is known, using weights to correct for heteroscedasticity is very simple using generalized least squares (GLS). If the form of heteroscedasticity is not known, the variance of each residual can be estimated first and these estimates can be used as weights in a second step and the resultant estimates are referred as estimated weighted least squares (EWLS) estimates. But in usual practice, the form of heteroscedasticity is seldom known, which makes the weighting approach impractical. In such situations, we are required to formulate such estimators those give as adequate results as if we have known heteroscedastic errors and hence adaptive estimation procedures come into play to fulfill such objectives. 
Specifically, in the sense of Bickel (1982), for a linear regression model in the presence of heteroscedasticity, an estimator is said to be adaptive estimator if it has the same asymptotic distribution as that of an estimator having information about the form of heteroscedasticity.

\subsection{Adaptive Nonparametric Kernel Estimator}

Carroll (1982) showed that even when the only thing known about the variances is that they are determined by an unknown but smooth function of the design or the mean response, the same asymptotic distribution as that of an estimator having information about the form of heteroscedasticity can be had. He followed the key idea noted by Fuller and Rao (1978) that in most of the heteroscedastic regression problems, the variances appeared to be smooth function of the design or mean response.

Consider the following heteroscedastic linear regression model:

$$
y_{i j}=x^{\prime}{ }_{i} \beta+u_{i j}, i=1,2, \ldots, m, j=1,2, \ldots, n,
$$

where $y_{i j}$ is the $j$ th response at the $i$ th design point $x_{i}, x_{i}$ are known $p$-vectors, $\beta$ is a $p$-vector of unknown parameters and $u_{i j}$ are the mutually independent with $\mathrm{E}\left(u_{i j}\right)=0$ and $\mathrm{E}\left(u_{i j}^{2}\right)=\sigma_{i}^{2}$. The variances $\sigma_{i}^{2}$ 's are unknown and heteroscedastic.

When the form of heteroscedasticity is not known, it is assumed that

$$
\sigma_{i}^{2}=g\left(x_{i}\right) \text { or } g\left(x_{i}^{\prime} \beta\right), \quad g(.) \text { unknown. }
$$

Carroll assumed variances as function of mean values as

$$
\sigma_{i}^{2}=g\left(x_{i}^{\prime} \beta\right)=g\left(\tau_{i}\right) \text {, where } g \text { is unknown, . }
$$

and defined the predicted values as

$$
t_{i}=x^{\prime}{ }_{i} \hat{\beta}_{\text {OLS }},
$$

where

$$
\hat{\beta}_{\text {OLS }}=\left(X^{\prime} X\right)^{-1} X^{\prime} y
$$

Carroll (1982), presented a kernel estimator of $g$ as

$$
\hat{g}_{N}(s)=\{N b(N)\}^{-1} \sum_{i=1}^{n} \sum_{j=1}^{m}\left(y_{i j}-\hat{y}_{i j}\right)^{2} K\left(\frac{t_{i}-s}{b(N)}\right)\left[\{N b(N)\}^{-1} \sum_{i=1}^{n} \sum_{j=1}^{m} K\left(\frac{t_{i}-s}{b(N)}\right)\right]^{-1},
$$

and the estimated variances are $\left\{\hat{g}_{N}\left(t_{i}\right)\right\}$. By using these estimated variances, an adaptive estimator can be devised as

$$
\hat{\beta}_{\text {AdK }}=\left(\sum_{i} X_{i} X^{\prime}{ }_{i} \hat{\sigma}_{i}^{-2}\right)^{-1} \sum_{i} X_{i} y_{i} \hat{\sigma}_{i}^{-2}
$$


In his paper, Carroll (1982) has shown that

$$
\operatorname{Asy} \operatorname{Var}\left\{n^{1 / 2}\left(\hat{\beta}_{A d K}-\beta\right)\right\}=\left(n^{-1} \sum_{i} X_{i} X_{i}^{\prime} \hat{\sigma}_{i}^{-2}\right)^{-1}
$$

\subsection{Nearest Neighbor Regression Estimator (NNRE)}

Similar to the results established by Carroll (1982) using kernel estimator under substantially more restrictive conditions on the data generating process, Robinson (1987) estimated the residual variances of unknown function of the explanatory variables by nearest neighbor nonparametric regression. The resulting weighted least squares estimator of the regression coefficient was shown to be adaptive, in the sense of having the same asymptotic distribution, to first order, as estimators based on knowledge of the actual variance function or a finite parameterization of it but under less restrictive conditions as compared to stated by Carroll(1982).

The adaptive NNRE can be computed as

$$
\hat{\beta}_{\text {AdNN }}=\left(\sum_{i} X_{i} X^{\prime}{ }_{i} \hat{\sigma}_{i}^{-2}\right)^{-1} \sum_{i} X_{i} Y_{i} \hat{\sigma}_{i}^{-2},
$$

where

$$
\hat{\sigma}_{i}^{2}=\sum_{j=1}^{n} e_{j}^{2} W_{i j}
$$

$e_{j}^{2}(j=1,2, \ldots, n)$ are squares of OLS residuals and $W_{i j}$ are the weights set by nearest nighbour approach( see Robinson, 1987 for more details).

\section{Money Demand Model}

We formulate Money Demand Model (see Gujarati, 2003 for similar formulation) for Pakistan as

where

$$
\ln M_{1 t}=\beta_{0}+\beta_{1} \ln G D P_{t}+\varepsilon_{t},
$$

$$
\begin{aligned}
& M_{1}=\text { Money Supply (in Millions of US Dollars) } \\
& G D P=\text { GDP at current market price (in Millions of US Dollars) }
\end{aligned}
$$

For the estimation of (3.1), we take annual available data, from 1975 to 2003 about Money Supply $\left(\mathrm{M}_{1}\right)$ and GDP.

\subsection{Estimation}

First of all we apply OLS to the data and then carry out White's test for heteroscedasticity (White, 1980). The test indicates a severe presence of heteroscedasticity with $p$-value 0.03 . Now as explained above, the correction of heteroscedasticity is mandatory to estimate the model (3.1).

We use both adaptive estimators, explained above, namely, kernel adaptive estimator and nearest neighbour regression estimator for the correction of heteroscedasticity of unknown form. For kernel estimation, we use normal kernel. 
For comparison purposes, we name the estimation used as

i) OLS : Ordinary least squares estimation

ii) AdK : Adaptive kernel estimation

iii) AdNN : Adaptive neighbour regression estimation

Fig. 3.1 shows the estimated form of heteroscedasticity using adaptive approach given by (2.2). This figure shows the estimated variances by both kernel and nearest neighbour regression methods. It should be noted for this figure that LGDP stands for Log GDP and E2i means square of OLS residuals. The presence of sever heteroscedasticity can also be assessed by Fig. 3.2 which shows heteroscedastic money supply.

In Table 3.1, we report about the performance of the three estimators OLS, AdK, AdNN, on the basis of standard errors of the estimated coefficients, standard error of regression, Akaike Information Criteria (AIC) value and the DurbanWatson (DW) statistic for autocorrelation. Expectedly, OLS shows poor performance in the presence of heteroscedasticity. It bears larger standard errors for the coefficients and same for the regression. We note that note that kernel estimator performs much better as compared to that of OLS showing lower standard errors. But AdNN outperforms the both of its competitors justifying the findings of Robinson (1987).

The OLS estimator shows higher AIC value while the other two have lower AIC showing the adequacy of the transformation of the model (3.1) in order to correct the heteroscedasticity and conducting weighted least squares. Again, AdNN has lowest AIC as compared to the rest two estimators.

Furthermore, we note the critical value from the Durbin-Watson table for testing autocorrelation. We find $d_{L}=1.34$ and report that AdNN, not only provides the suitable weights for correcting heteroscedasticity but also tries to remove the effect of autocorrelation. This is because the DW statistic provided by AdNN comes close to $d_{L}$.
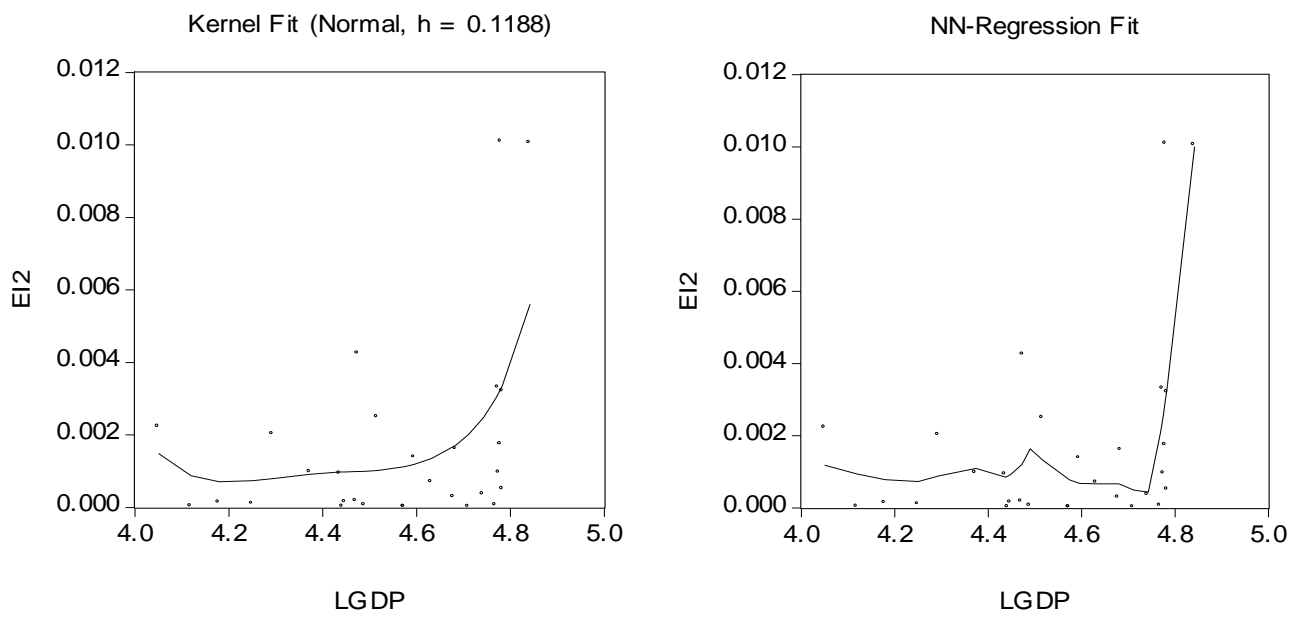

Fig. 3.1: Estimated Heteroscedastic Pattern by Adaptive Approach 


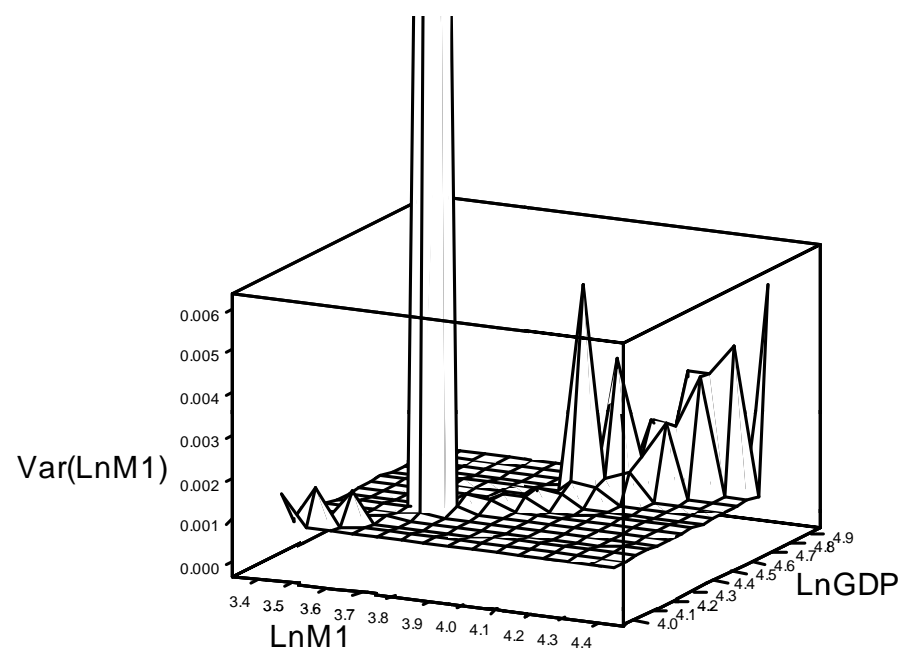

Fig. 3.2: Heteroscedastic Money Supply $\left(M_{1}\right)$

Table 3.1: Estimates of Money-Demand Model of Pakistan (1975-2003)

\begin{tabular}{|c|c|c|c|c|c|c|c|c|c|}
\hline \multirow[b]{2}{*}{ Estimators } & \multicolumn{3}{|c|}{ Estimation of $\beta_{0}$} & \multicolumn{3}{|c|}{ Estimation of $\beta_{1}$} & \multirow{2}{*}{$\begin{array}{c}\text { S.E. of } \\
\text { Regression }\end{array}$} & \multirow[b]{2}{*}{ AIC } & \multirow[b]{2}{*}{ DW } \\
\hline & $\hat{\beta}_{0}$ & S.E & $\begin{array}{c}\mathrm{t}- \\
\text { statistic }\end{array}$ & $\hat{\beta}_{1}$ & S.E & $\begin{array}{c}\mathrm{t}- \\
\text { statistic }\end{array}$ & & & \\
\hline OLS & -0.761 & 0.1635 & -4.66 & 1.042 & 0.0359 & 29.06 & 0.0420 & -3.44 & 0.966 \\
\hline AdK & -0.730 & 0.1411 & -5.17 & 1.036 & 0.0316 & 32.80 & 0.0355 & -3.77 & 1.188 \\
\hline AdNN & -0.789 & 0.1270 & -6.21 & 1.049 & 0.0280 & 37.42 & 0.0324 & -3.95 & 1.214 \\
\hline
\end{tabular}

\section{Conclusion}

Money supply usually shows heteroscedastic pattern. But for most of the practical situations, we seldom know any thing about the form of heteroscedasticity then it becomes a question to correct the analysis for heteroscedasticity. When the form of heteroscedasticity is unknown, we may use adaptive estimators that give desirable results that are not possible when just OLS estimation is taken into account. Usually nonparametric approaches are used for adaptive estimation. In these approaches nearest neighbour regression estimators give better results as compared to adaptive kernel estimators in the presence of heteroscedasticity of unknown form.

\section{References}

1. Ahmed, N., Shah, H., Agha, A. I., and Mubarik, Y. A. (2005). Transmission Mechanism of Monetary Policy in Pakistan. Working Papers No. 9, State Bank of Pakistan.

2. Bickel, P. J. (1982). On Adaptive Estimation. Annals of Statistics, 10, 647-671. 
3. Carroll, R. J. (1982). Adapting for Heteroscedasticity in Linear Models. Annals of Statistics, 10, 1224-1233.

4. Fuller, W. A. and Rao, J. N. K. (1978). Estimation for a Linear Regression Model with Unknown Diagonal Covariance Matrix. Annals of Statistics, 6, 1149-1158.

5. Geary, R. C. (1966). A Note on residual Heretovariance and Estimation Efficiency in Regression. American Statistician, 20, 30-31.

6. Griffiths, W. E. (1999). Heteroskedasticity. Working Papers in Eco. \& App. Stat. ISSN 0157-0188.

7. Gujarati, D. N. (2003). Basic Econometrics. 4th Ed., McGraw-Hill, New York.

8. Hossain, A. (1994). Time Series Properties of Variables in the Money Demand Function in Pakistan. Indian Economic Review, 29, 195-210.

9. Khan, M. and Hussain, F. (2005). Monetary Aggregates in Pakistan: Theoretical and Empirical Underpinnings. Working Papers No. 7, State Bank of Pakistan.

10. Pasha, G. R. (1982). Estimation Methods for Regression Models with Unequal Error Variances. University of Warwick, Ph.D. Thesis.

11. Robinson, P. M. (1987). Asymptotically Efficient Estimation in the Presence of Heteroskedasticity of Unknown Form. Econometrica, 55, 875-891.

12. White, H. (1980). A Heteroksedasticity-Consistent Covariance Matrix Estimator and Direct Test for Heteroskedasticity. Econometrica, 48, 817-838. 